\title{
PROBABILISTIC RESULTS FOR A MOBILE SERVICE SCENARIO
}

\author{
JESPER MØLLER, * Aalborg University \\ MAN LUNG YIU,** Hong Kong Polytechnic University
}

\begin{abstract}
We consider the following stochastic model for a mobile service scenario. Consider a stationary Poisson process in $\mathbb{R}^{d}$, with its points radially ordered with respect to the origin (the anchor); if $d=2$, the points may correspond to locations of, e.g. restaurants. A user, with a location different from the origin, asks for the location of the first Poisson point and keeps asking for the location of the next Poisson point until the first time that he/she can be completely certain that he/she knows which Poisson point is his/her nearest neighbour. This waiting time is the communication cost, while the inferred privacy region is a random set obtained by an adversary who only knows the anchor and the points received from the server, where the adversary 'does the best' to infer the possible locations of the user. Probabilistic results related to the communication cost and the inferred privacy region are established for any dimension $d \geq 1$. Furthermore, special results when $d=1$ and particularly when $d=2$ are derived.
\end{abstract}

Keywords: Communication cost; nearest-neighbour search; Poisson process; privacy region; radial simulation algorithm; Voronoi tessellation

2010 Mathematics Subject Classification: Primary 60D05; 60G55; 62M30

Secondary $68 \mathrm{U} 20$

\section{Introduction}

The mobile Internet offers services that, e.g. receive the location of the nearest point of interest such as a store, restaurant, or tourist attraction; see [9] and the references therein. This paper demonstrates that tools from applied probability and in particular stochastic geometry can be useful when analysing the performance of such services.

In this paper we consider a setting for a mobile service protocol proposed in [9], where a user is located at a point $q \in \mathbb{R}^{d}$ and a stationary Poisson point process $\Phi=\left\{X_{1}, X_{2}, \ldots\right\} \subset \mathbb{R}^{d}$ is given; for the problem setting in [9], $d=2$ and the points in $\Phi$ may, e.g. correspond to the locations of stores. In order to preserve some privacy, the user queries a server for nearby points in $\Phi$ but he/she reports not his/her correct location $q$ but another location $q^{\prime} \in \mathbb{R}^{d}$ referred to as the anchor. An incremental query processing on the server is used so that the points $X_{1}, X_{2}, \ldots$ are ordered in increasing distance to the anchor. The user then stops to query the server as soon as possible, i.e. when the nearest point in $\Phi$ with respect to $q$ can be determined. The waiting time for this to happen is called the communication cost and is denoted by $M$. Another object of interest is the inferred privacy region, which is a random set $\mathcal{R} \subset \mathbb{R}^{d}$ obtained by an adversary

Received 4 May 2010; revision received 10 December 2010.

* Postal address: Department of Mathematical Sciences, Aalborg University, Fredrik Bajers Vej 7G, DK-9220 Aalborg, Denmark. Email address: jm@math.aau.dk

** Postal address: Department of Computing, Hong Kong Polytechnic University, Hung Hom, Kowloon, Hong Kong. Email address: csmlyiu@comp.polyu.edu.hk 
who knows only the location of the anchor and the points received from the server, where the adversary 'does the best' to infer the possible locations of the user. The precise definitions of $M$ and $\mathcal{R}$ are given in Sections 2 and 3, respectively.

The assumption that $\Phi$ is a stationary Poisson process is motivated by the fact that this the most fundamental spatial point process model in stochastic geometry, and it often serves as a natural starting point for statistical analysis; see, e.g. [1], [4], and [8]. Our objective is to analyse the distribution of $M$ and various properties of $\mathcal{R}$, where we exploit the independence properties of the Poisson process to derive analytical results; for other point process models, Monte Carlo simulations will probably be needed. In Section 2, the distribution and moments for $M$ are derived in detail. In Section 3 we describe first the geometric properties of $\mathcal{R}$, and then establish results related to the probability that $\mathcal{R}$ contains a given point in $\mathbb{R}^{d}$ and the expected value of $V$, where $V=|\mathcal{R}|$ is the $d$-dimensional volume of the inferred privacy region.

\section{The communication cost}

\subsection{Preliminaries}

2.1.1. Assumptions. Throughout the sequel, it may be assumed without loss of generality that $q^{\prime}=o$, the origin in $\mathbb{R}^{d}$; we will keep using the notation $q^{\prime}$ in order to remind the reader that this refers to the anchor. Denote by $l=\left\|q-q^{\prime}\right\|$ the distance between the anchor and the user location, and by $R_{i}=\left\|X_{i}-q^{\prime}\right\|$ the distance of $X_{i}$ to the anchor. The case where $l=0$ turns out to be trivial since $\Phi$ is a stationary Poisson process, so we assume that $l>0$. Any point $X_{i}$ in $\Phi$ is a random variable, and we order the points in $\Phi$ such that $0 \leq R_{1} \leq R_{2} \leq \cdots$. Note that these inequalities are strict almost surely. Denote by $Z$ the nearest neighbour to $q$ among $X_{1}, X_{2}, \ldots$. For $i=1,2, \ldots$, let $Q_{i}$ be the nearest neighbour to $q$ among the first $i$ points $X_{1}, \ldots, X_{i}$, and set $D_{i}=\left\|q-Q_{i}\right\|$ (so $Z$ and $Q_{i}$ are almost surely uniquely defined). Denote by

$$
B(x, r)=\left\{y \in \mathbb{R}^{d}:\|y-x\| \leq r\right\}
$$

the closed ball in $\mathbb{R}^{d}$ with centre $x \in \mathbb{R}^{d}$ and radius $r \geq 0$. Let $|\cdot|$ denote the volume (Lebesgue measure) in $\mathbb{R}^{d}$, and let

$$
\omega_{d}=|B(0,1)|=\frac{\pi^{d / 2}}{\Gamma(1+d / 2)}
$$

be the volume of the $d$-dimensional unit ball, where $\Gamma$ is the gamma function. Finally, denote by $\rho>0$ the intensity of $\Phi$ and define

$$
\alpha=(\rho|B(0, l)|)^{1 / d}=\left(\omega_{d} \rho\right)^{1 / d} l .
$$

2.1.2. Definition of the communication cost. Denote by $\mathbb{N}=\{1,2, \ldots\}$ the set of positive integers. For $i \in \mathbb{N}$, define the demand space by $\mathscr{D}_{i}=B\left(q, D_{i}\right)$ and the supply space by $\delta_{i}=B\left(q^{\prime}, R_{i}\right)$. Then $\left(D_{i}\right)_{i \in \mathbb{N}}$ is decreasing, $\left(\delta_{i}\right)_{i \in \mathbb{N}}$ is increasing, and we define the communication cost as the discrete random variable $M$ given by the first time the demand space is included in the supply space, that is,

$$
M=\inf \left\{i \in \mathbb{N}: \mathscr{D}_{i} \subseteq \wp_{i}\right\}
$$

(setting inf $\varnothing=\infty$ ). See Figure 1. In other words, for any $m \in \mathbb{N}, M=m$ if $m$ is the first time the user can be completely ensured that $Z=Q_{m}$ when he/she has only received $X_{1}, \ldots, X_{m}$ from the server, i.e. no matter where the points $X_{m+1}, X_{m+2}, \ldots$ could potentially be located 


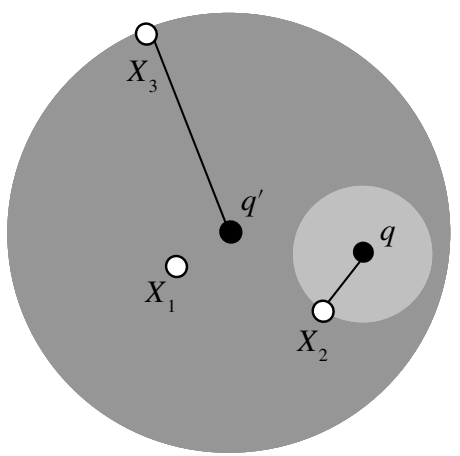

Figure 1: A planar example $(d=2)$ with $M=3$, showing the corresponding demand space $\mathscr{D}_{3}$ and supply space $\varsigma_{3}$. Note that $\mathscr{D}_{2}=\mathscr{D}_{3}$ and $Z=Q_{2}=Q_{3}$.

in $\mathbb{R}^{d} \backslash B\left(q^{\prime}, R_{m}\right)$. Note that $M$ is almost surely finite (this is verified in Lemma 1 below), in which case

$$
Z=Q_{M}
$$

is returned as the nearest neighbour to $q$.

\subsection{Results}

For $d \geq 2$, we can exclude certain events of zero probability and thereby simplify the meaning of $M$ and $Z$ as stated in the following lemma.

Lemma 1. For $d \geq 2$, with probability $1, M$ is finite and given by the smallest integer $m \geq 2$ such that $D_{m-1}$ is a strict subset of $S_{m}$, and

$$
Z=Q_{M}=Q_{M-1}, \quad D_{M}=D_{M-1}, \quad R_{M} \geq l .
$$

Proof. Clearly, by (1), $R_{M} \geq l$. Since $M=1$ implies that $X_{1}$ lies on the half-line $H$ with endpoint $q$ and direction $q-q^{\prime}$, and this happens with probability 0 , we have, almost surely, $M \geq 2$. Furthermore, with probability 1 , for $m \in\{2,3, \ldots\}, M=m$ implies that $Q_{m} \neq X_{m}$ because $Q_{m}=X_{m}$ would imply that $X_{m} \in H$, which happens with probability 0 . Moreover, with probability 1 , the sequence $R_{1}, R_{2}, \ldots$ is strictly increasing to $\infty$, and so the sequence of supply spaces $\varsigma_{1} \subset \varsigma_{2} \subset \cdots$ tends to $\mathbb{R}^{d}$. On the other hand, the sequence of demand spaces decreases. Combining these facts with (1) and (2), the assertions of the lemma are seen to be true.

Let $T=\|Z-q\|$ be the distance from the user to its nearest point in $\Phi$, and let $\Psi=$ $\Phi \cap\left[B\left(q^{\prime}, T+l\right) \backslash B(q, T)\right]$ be the restriction of $\Phi$ to the random set $B\left(q^{\prime}, T+l\right) \backslash B(q, T)$. Let $N$ denote the number of points in $\Psi$, and set $S=\omega_{d} \rho T^{d}$ and

$$
\Lambda=\rho\left|B\left(q^{\prime}, l+T\right) \backslash B\left(q^{\prime}, T\right)\right|=\left(\alpha+S^{1 / d}\right)^{d}-S=\sum_{i=0}^{d-1}\left(\begin{array}{l}
d \\
i
\end{array}\right) \alpha^{d-i} S^{i / d} .
$$

Since $\Phi$ is a Poisson process, then

(i) $S$ is exponentially distributed with parameter 1 ;

(ii) conditional on $Z, \Phi \backslash B(q, T)$ is a homogeneous Poisson process on $\mathbb{R}^{d} \backslash B(q, T)$ with intensity $\rho$, and $\Psi$ is a homogeneous Poisson process on $B\left(q^{\prime}, T+l\right) \backslash B(q, T)$ with its mean number of points equal to $\Lambda$; 
(iii) in the special case $d=1$, the event $Z=X_{M}$ is equivalent to the event that $Z-q$ has the same sign as $q-q^{\prime}$, so $\mathrm{P}\left(Z=X_{M}\right)=\frac{1}{2}$, and if $Z=X_{M}$ then $N=M-1$ and $\Psi=$ $\left\{X_{1}, \ldots, X_{M-1}\right\}$, while if $Z \neq X_{M}$ then $N=M-2$ and $\Psi=\left\{X_{1}, \ldots, X_{M-1}\right\} \backslash\{Z\}$;

(iv) for $d \geq 2$, with probability $1, N=M-2$ and $\Psi=\left\{X_{1}, \ldots, X_{M-1}\right\} \backslash\{Z\}$; cf. Lemma 1 . These results are now used to obtain the distribution of $N$ (or, equivalently, $M$ ), where $\operatorname{Po}(\alpha)$ denotes the Poisson distribution with parameter $\alpha$.

Theorem 1. (a) For $d=1, N$ is independent of $Z$ and follows $\operatorname{Po}(\alpha)$,

$$
\mathrm{P}(M=1)=\frac{\mathrm{e}^{-\alpha}}{2}, \quad \mathrm{P}(M=m)=\left(\frac{\alpha^{m-1}}{(m-1) !}+\frac{\alpha^{m-2}}{(m-2) !}\right) \frac{\mathrm{e}^{-\alpha}}{2}, \quad m=2,3, \ldots,
$$

and $M$ has mean and variance

$$
\mathrm{E}(M)=\alpha+\frac{3}{2}, \quad \operatorname{var}(M)=\alpha+\frac{1}{4},
$$

with $\alpha=2 \rho l$.

(b) For $d=2$,

$$
\mathrm{P}(M=m)=\int_{0}^{\infty} \frac{\left(\alpha^{2}+2 \alpha \sqrt{s}\right)^{m-2}}{(m-2) !} \mathrm{e}^{-\left(\alpha^{2}+2 \alpha \sqrt{s}+s\right)} \mathrm{d} s, \quad m=2,3, \ldots,
$$

and

$$
\mathrm{E}(M)=\alpha^{2}+\sqrt{\pi} \alpha+2, \quad \operatorname{var}(M)=(5-\pi) \alpha^{2}+\sqrt{\pi} \alpha,
$$

with $\alpha=\sqrt{\pi \rho} l$.

(c) For $d \geq 2, M-2$ conditional on $Z$ follows $\operatorname{Po}(\Lambda)$, and

$$
\begin{aligned}
\mathrm{E}(M)= & 2+\sum_{i=0}^{d-1}\left(\begin{array}{l}
d \\
i
\end{array}\right) \alpha^{d-i} \Gamma\left(1+\frac{i}{d}\right), \\
\operatorname{var}(M)= & \sum_{i=0}^{d-1}\left(\begin{array}{l}
d \\
i
\end{array}\right) \alpha^{d-i} \Gamma\left(1+\frac{i}{d}\right) \\
& +\sum_{i=1}^{d-1} \sum_{j=1}^{d-1}\left(\begin{array}{l}
d \\
i
\end{array}\right)\left(\begin{array}{l}
d \\
j
\end{array}\right) \alpha^{2 d-i-j}\left[\Gamma\left(1+\frac{i+j}{d}\right)-\Gamma\left(1+\frac{i}{d}\right) \Gamma\left(1+\frac{j}{d}\right)\right] .
\end{aligned}
$$

(d) For $d \geq 1$ and any number $\beta, \mathrm{E}\left(M^{\beta}\right)<\infty$.

Proof. If $d=1$, since $\Lambda=\alpha$ is then deterministic, (ii) implies that $N$ is independent of $Z$ and follows $\operatorname{Po}(\alpha)$. Hence, (iii) easily implies (4) and (5), and so (a) follows.

If $d \geq 2$ then, by (ii), $N$ conditional on $Z$ follows $\operatorname{Po}(\Lambda$ ), and, by (iv), $M=N+2$, so $\mathrm{E}(M)=2+\mathrm{E}(\Lambda)$ and

$$
\operatorname{var}(M)=\operatorname{var}(N)=\mathrm{E}(\operatorname{var}(N \mid \Lambda))+\operatorname{var}(\mathrm{E}(N \mid \Lambda))=\mathrm{E}(\Lambda)+\operatorname{var}(\Lambda) .
$$

Combining this with (3) and the fact that, by (i), $\mathrm{E}\left(S^{\beta}\right)=\Gamma(\beta+1)$ for $\beta>-1$, we find, after a straightforward calculation, that (8) and (9) hold for $d \geq 2$, where (7) is the case with 
$d=2$. Then (6) immediately follows by combining (3), (i), and the fact that $N$ conditional on $S$ follows $\operatorname{Po}(\Lambda)$. Thereby, (b)-(c) are verified.

For $\beta \leq 0, M^{\beta} \leq 1$, and so (d) clearly holds. For $\beta>0$, (d) follows immediately from (a) if $d=1$, and from (c) and (3) if $d \geq 2$, using again the fact that $\mathrm{E}\left(S^{\beta}\right)=\Gamma(\beta+1)$. Hence, (d) is verified.

Suppose that $d=2$, and let $\operatorname{erf}(\alpha)=(2 / \sqrt{\pi}) \int_{0}^{\alpha} \exp \left(-t^{2}\right) \mathrm{d} t$ be the 'error function'. Then (6) gives

$$
\mathrm{P}(M=2)=\exp \left(-\alpha^{2}\right)+\alpha \sqrt{\pi}(\operatorname{erf}(\alpha)-1)
$$

which strictly decreases from 1 to 0 as $\alpha$ increases from 0 to $\infty$. We have also evaluated the integral in (6) for $m=3,4, \ldots$ using the computational software program MAPLE ${ }^{\circledR}$, but, since the number of terms increases fast as $m$ increases, we omit the results here. By (3) and Theorem 1(c), $M-2$ conditional on $S$ follows $\operatorname{Po}\left(\alpha^{2}+2 \alpha \sqrt{S}\right)$. Hence, as $\alpha \rightarrow \infty, M / \alpha-\alpha$ converges in distribution to a mixture of normal distributions with mean $2 \sqrt{S}$ and unit variance.

\section{The inferred privacy region}

\subsection{Preliminaries}

3.1.1. Definition of the inferred privacy region. Suppose that an adversary knows the location $q^{\prime}$ of the anchor, the termination conditions (1)-(2), the termination time $M$, and the points $X_{1}, \ldots, X_{M}$ received from the server, while the location $q$ of the user is unknown to him/her. If the adversary then wants to infer the possible locations of $q$, the best the adversary can do is to estimate $q$ to be contained in the inferred privacy region which is a random set $\mathcal{R}$ specified below.

Consider the Voronoi tessellation of $\mathbb{R}^{d}$ generated by $\left\{X_{1}, \ldots, X_{M}\right\}$, with cells

$$
C_{i}=\left\{x \in \mathbb{R}^{d}:\left\|x-X_{i}\right\| \leq\left\|x-X_{j}\right\|, j=1, \ldots, M\right\}, \quad i=1, \ldots, M .
$$

The Voronoi cells have disjoint interiors with boundaries of zero volume (with respect to Lebesgue measure in $\mathbb{R}^{d}$ ), and, with probability 1 , they are $d$-dimensional sets [3], [5]. Note that $B\left(x,\left\|x-X_{i}\right\|\right) \subseteq B\left(q^{\prime}, r\right)$ if and only if $\left\|x-q^{\prime}\right\|+\left\|x-X_{i}\right\| \leq r$. Consequently, if $M \geq 2$ and $i \in\{1, \ldots, M-1\}$, the set

$$
E_{i}=\left\{x \in C_{i}: R_{M-1}<\left\|x-q^{\prime}\right\|+\left\|x-X_{i}\right\| \leq R_{M}\right\}
$$

consists 'essentially' of all possible locations $x$ of the user such that $X_{i}$ is returned under the termination conditions as the nearest neighbour to $x$. By 'essentially' we mean that if $x$ is on the boundary of $C_{i}$ so that $x \in C_{j}$ for some $j<i$, then $X_{i}$ would not have been returned, but the set of such points $x$ has zero volume and, as argued in comment (E) below, it plays no important role but is just convenient that we have included such points in $E_{i}$. Moreover, the set of all possible locations $x \in C_{M} \backslash \bigcup_{i=1}^{M-1} C_{i}$ of the user is given by

$$
E_{M}=\left[q^{\prime}, X_{M}\right] \backslash \bigcup_{i=1}^{M-1} C_{i},
$$

where $\left[q^{\prime}, X_{M}\right]$ is the closed line segment with end points $q^{\prime}$ and $X_{M}$, and we set $\bigcup_{i=1}^{M-1} C_{i}=\varnothing$ if $M=1$. The inferred privacy region is therefore given by

$$
\mathcal{R}=\bigcup_{i=1}^{M} E_{i} .
$$




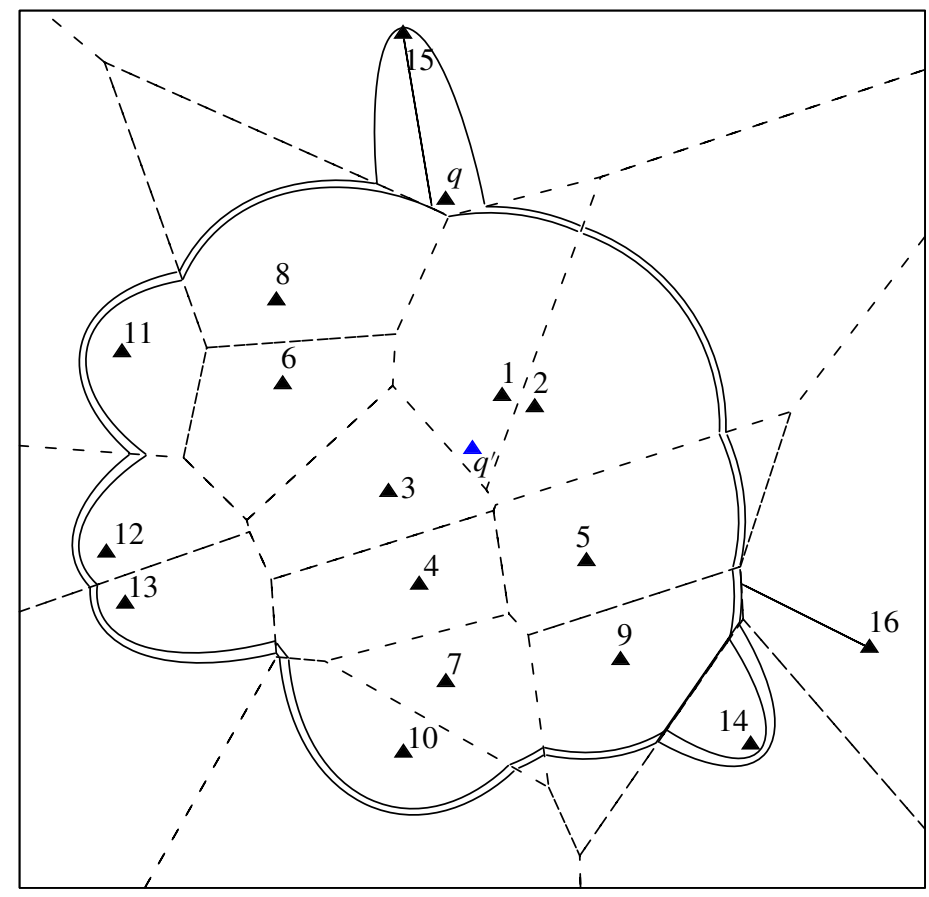

FIGURE 2: A planar inferred privacy region $\mathcal{R}$ (the solid lines except those on the boundary of the square) defined by $M=16$ points (the triangles marked $1, \ldots, 16$ ), and the anchor $q^{\prime}$. The Voronoi tessellation with nuclei $X_{1}, \ldots, X_{M}$ is shown as dashed lines. Also, the user location $q$ is shown, but the inferred privacy region is unchanged if any other point in $\mathcal{R}$ had been the location of the user.

Figure 2 shows an example of $\mathcal{R}$ when $d=2$ and the Poisson points are generated by the conventional radial simulation algorithm due to Quine and Watson [6].

3.1.2. Comments. Some remarks are in order.

(A) If $M=1$ then simply $\mathcal{R}=E_{M}=\left[q^{\prime}, X_{M}\right]$.

(B) If $1 \leq i \leq M-1$ then by (10), $E_{i}=C_{i} \cap\left(F_{i} \backslash G_{i}\right)$, where $F_{i}$ and $G_{i}$ are the ellipsoidal regions with foci $q^{\prime}$ and $X_{i}$, such that any point on the boundary has its sum of distances to the foci equal to $R_{M}$ and $R_{M-1}$, respectively (see cells $1, \ldots, 15$ in Figure 2). As illustrated in Figure 2, $E_{i}$ can be a connected set (see, e.g. cell 1) or a disconnected set (see cell 9) or the empty set (see, e.g. cell 3), $G_{M-1}=\left[q^{\prime}, X_{M-1}\right]$ is a closed line segment (see cell 15), while, for $1 \leq i \leq M-2, F_{i}$ and $G_{i}$ are almost surely of dimension $d$.

(C) If $M \geq 2$ then $E_{M}$ is the line segment given by the intersection of $\left[q^{\prime}, X_{M}\right]$ and the interior of $C_{M}$ (see cell 16 in Figure 2); cf. (11).

(D) If $d \geq 2, E_{M}$ has zero $d$-dimensional volume, and the adversary could exclude both the possibility that $M=1$ and the possibility that $X_{M}$ is the nearest point in $\Phi$ to the user, since the event that $M=1$ or $x \in\left[q, X_{M}\right]$ has probability 0 .

(E) Recalling the considerations in connection to (10) concerning the boundary points of the Voronoi cells, note that $\mathcal{R}=\bigcup_{i=1}^{M} E_{i}^{\prime}$, where $E_{i}^{\prime}=E_{i} \backslash \bigcup_{j=1}^{i-1} C_{j}$ is exactly the set of all 
possible locations $x$ of the user such that $X_{i}$ is returned under the termination conditions as the nearest neighbour to $x$ (setting $\bigcup_{j=1}^{i-1} C_{j}=\varnothing$ if $i=1$ ). Clearly, $E_{i} \backslash E_{i}^{\prime}$ has zero volume.

\subsection{Representation formula}

The volume of the inferred privacy region, $V=|\mathcal{R}|$, is a 'measure of privacy' with mean value

$$
\mathrm{E}(V)=\int p(x) \mathrm{d} x
$$

where, for any location $x \in \mathbb{R}^{d}$,

$$
p(x)=\mathrm{P}(x \in \mathcal{R})
$$

is the probability that $x$ is in the inferred privacy region. Writing $Z=q+T U$, then $U$ is a uniformly distributed unit vector in $\mathbb{R}^{d}, T$ and $U$ are independent, and $T^{d}$ is exponentially distributed with rate $\rho \omega_{d}$, so $Z$ has density function

$$
f(z)=\rho \exp \left(-\rho \omega_{d}\|z-q\|^{d}\right) .
$$

Our strategy is first to determine the conditional probability

$$
p(x \mid z)=\mathrm{P}(x \in \mathcal{R} \mid Z=z),
$$

second to calculate

$$
p(x)=\int p(x \mid z) f(z) \mathrm{d} z,
$$

and, finally, to obtain $\mathrm{E}(V)$ from (13).

In the remainder of this paper, we focus on the case $d \geq 2$. Theorem 2 below establishes an expression for $p(x \mid z)$; similar techniques apply in the special case $d=1$, but the details are then somewhat more complicated since we have to account for each of the cases where $Z=X_{M}$ and $Z \neq X_{M}$; cf. (iii) (above Theorem 1).

Let $\mathbf{1}[\cdot]$ denote the indicator function. For $x, y \in \mathbb{R}^{d}$ and $r, s, t \geq 0$, define

$$
c(r, s, t)=|B(x, s) \cap B(y, t)| \quad \text { if } r=\|x-y\|
$$

and

$$
\begin{aligned}
& p(x, y, t)=\mathbf{1}\left[\left\|x-q^{\prime}\right\|+\|y-x\| \geq t+l\right] \\
& \times \exp \left(-\rho\left\{\omega_{d}\left[\left(\left\|x-q^{\prime}\right\|+\|y-x\|\right)^{d}-(t+l)^{d}\right]\right.\right. \\
& \left.\left.\quad-c\left(\left\|x-q^{\prime}\right\|, t+l,\|y-x\|\right)+c(\|x-q\|, t,\|y-x\|)\right\}\right) \\
& +\mathbf{1}\left[\left\|x-q^{\prime}\right\|+\|y-x\|<t+l\right] \\
& \times \exp \left(-\rho\left\{\omega_{d}\left[(t+l)^{d}-t^{d}-\left(\left\|x-q^{\prime}\right\|+\|y-x\|\right)^{d}+\|y-x\|^{d}\right]\right.\right. \\
& \left.\left.\quad+c\left(l, t,\left\|x-q^{\prime}\right\|+\|y-x\|\right)-c(\|x-q\|, t,\|y-x\|)\right\}\right) .
\end{aligned}
$$

Note that $c(r, s, t)=0$ if $r \geq s+t$ or $s=0$ or $t=0$, and $c(r, s, t)=\omega_{d} \min \left\{s^{d}, t^{d}\right\}$ if $r=0$. If $d=2, r<s+t$, and $r, s, t>0$, then

$$
\begin{aligned}
c(r, s, t)= & s^{2} \arccos \left(\frac{r^{2}+s^{2}-t^{2}}{2 r s}\right)-\frac{r^{2}+s^{2}-t^{2}}{4 r^{2}}\left[4 r^{2} s^{2}-\left(r^{2}+s^{2}-t^{2}\right)^{2}\right]^{1 / 2} \\
& +t^{2} \arccos \left(\frac{r^{2}+t^{2}-s^{2}}{2 r t}\right)-\frac{r^{2}+t^{2}-s^{2}}{4 r^{2}}\left[4 r^{2} t^{2}-\left(r^{2}+t^{2}-s^{2}\right)^{2}\right]^{1 / 2} .
\end{aligned}
$$


Theorem 2. For $d \geq 2$ and $x, z \in \mathbb{R}^{d}$, letting $t=\|z-q\|$, then

$$
\begin{aligned}
& p(x \mid z)=p(x, z, t) \\
& +\rho \int \mathbf{1}\left[y \in B\left(q^{\prime}, t+l\right) \backslash B(q, t),\right. \\
& \left.\quad z \in B\left(q^{\prime},\left\|x-q^{\prime}\right\|+\|y-x\|\right) \backslash B(x,\|y-x\|)\right] p(x, y, t) \mathrm{d} y .
\end{aligned}
$$

Proof. We start by verifying that

$$
p(x, y, t)=p_{1}(x, y, t) p_{2}(x, y, t),
$$

where

$$
p_{1}(x, y, t)=\exp \left(-\rho \omega_{d} \max \left\{0,\left(\left\|x-q^{\prime}\right\|+\|y-x\|\right)^{d}-(t+l)^{d}\right\}\right)
$$

and

$$
\begin{aligned}
& p_{2}(x, y, t) \\
& \quad=\exp \left(-\rho\left|\left\{B\left(q^{\prime}, t+l\right) \backslash B(q, t)\right\} \backslash\left\{B\left(q^{\prime},\left\|x-q^{\prime}\right\|+\|y-x\|\right) \backslash B(x,\|y-x\|)\right\}\right|\right) .
\end{aligned}
$$

Note that

$$
\begin{aligned}
p_{1}(x, y, t) p_{2}(x, y, t)= & \mathbf{1}\left[\left\|x-q^{\prime}\right\|+\|y-x\| \geq t+l\right] p_{1}(x, y, t) p_{2}(x, y, t) \\
& +\mathbf{1}\left[\left\|x-q^{\prime}\right\|+\|y-x\|<t+l\right] p_{2}(x, y, t) .
\end{aligned}
$$

If $\left\|x-q^{\prime}\right\|+\|y-x\| \geq t+l$ then $B(q, t) \subseteq B\left(q^{\prime}, t+l\right) \subseteq B\left(q^{\prime},\left\|x-q^{\prime}\right\|+\|y-x\|\right)$, and so

$$
p_{2}(x, y, t)=\exp \left(-\rho\left[c\left(\left\|x-q^{\prime}\right\|, t+l,\|y-x\|\right)-c(\|x-q\|, t,\|y-x\|)\right]\right) .
$$

If $\left\|x-q^{\prime}\right\|+\|y-x\|<t+l$ then $B(x,\|y-x\|) \subseteq B\left(q^{\prime},\left\|x-q^{\prime}\right\|+\|y-x\|\right) \subseteq B\left(q^{\prime}, t+l\right)$ and $B(q, t) \subseteq B\left(q^{\prime}, t+l\right)$, and so if $A^{\mathrm{c}}=\mathbb{R}^{d} \backslash A$ denotes the complement of a set $A \subseteq \mathbb{R}^{d}$,

$$
\begin{aligned}
\left|\left\{B\left(q^{\prime}, t+l\right) \backslash B(q, t)\right\} \backslash\left\{B\left(q^{\prime},\left\|x-q^{\prime}\right\|+\|y-x\|\right) \backslash B(x,\|y-x\|)\right\}\right| \\
=\left|B\left(q^{\prime}, t+l\right) \cap B(q, t)^{\mathrm{c}} \cap\left[B\left(q^{\prime},\left\|x-q^{\prime}\right\|+\|y-x\|\right)^{\mathrm{c}} \cup B(x,\|y-x\|)\right]\right| \\
=\left|B\left(q^{\prime}, t+l\right) \cap B(q, t)^{\mathrm{c}} \cap B\left(q^{\prime},\left\|x-q^{\prime}\right\|+\|y-x\|\right)^{\mathrm{c}}\right| \\
\quad+\left|B\left(q^{\prime}, t+l\right) \cap B(q, t)^{\mathrm{c}} \cap B(x,\|y-x\|)\right| \\
=\left[\left|B\left(q^{\prime}, t+l\right)\right|-|B(q, t)|-\left|B\left(q^{\prime},\left\|x-q^{\prime}\right\|+\|y-x\|\right)\right|\right. \\
\left.\quad+\left|B(q, t) \cap B\left(q^{\prime},\left\|x-q^{\prime}\right\|+\|y-x\|\right)\right|\right] \\
\quad+[|B(x,\|y-x\|)|-|B(q, t) \cap B(x,\|y-x\|)|] .
\end{aligned}
$$

Now, (18) follows from (16) and (19)-(21).

Denote by $Z(x)$ the almost surely unique nearest point to $x$ in $\left\{X_{1}, \ldots, X_{M}\right\}=\Psi \cup\left\{Z, X_{M}\right\}$ (so $Z(q)=Z$ ). By (10)-(12) and (D) in Section 3.1.2,

$$
\begin{aligned}
\mathrm{P}(x \in \mathcal{R} \mid Z)= & \mathrm{P}\left(Z=Z(x), R_{M-1}<\left\|x-q^{\prime}\right\|+\|Z-x\| \leq R_{M} \mid Z\right) \\
& +\mathrm{E}\left(\sum_{i=1}^{N} \mathbf{1}\left[X_{i}=Z(x), R_{M-1}<\left\|x-q^{\prime}\right\|+\left\|X_{i}-x\right\| \leq R_{M}\right] \mid Z\right) .
\end{aligned}
$$


By (iv) (above Theorem 1), with probability 1,

$$
Z=Z(x) \quad \Longleftrightarrow \quad\left[\Psi \cup\left\{X_{M}\right\}\right] \cap B(x,\|Z-x\|)=\varnothing
$$

Conditional on $Z, R_{M}^{d}-(T+l)^{d}$ is exponentially distributed and independent of $\Psi$; cf. (ii) (above Theorem 1). Therefore, ignoring the null set where

$$
\left\|Z-q^{\prime}\right\|=\left\|x-q^{\prime}\right\|+\|Z-x\|
$$

(i.e. $\left\|Z-q^{\prime}\right\|<\left\|x-q^{\prime}\right\|+\|Z-x\|$ almost surely),

$$
\begin{aligned}
\mathrm{P}(Z= & \left.Z(x), R_{M-1}<\left\|x-q^{\prime}\right\|+\|Z-x\| \leq R_{M} \mid Z\right) \\
= & \mathrm{P}\left(\left\|x-q^{\prime}\right\|+\|Z-x\| \leq R_{M}, X_{M} \notin B(x,\|Z-x\|) \mid Z\right) \\
& \times \mathrm{P}\left(\Psi \subset B\left(q^{\prime},\left\|x-q^{\prime}\right\|+\|Z-x\|\right) \backslash B(x,\|Z-x\|) \mid Z\right) \\
= & p_{1}(x, Z, T) p_{2}(x, Z, T),
\end{aligned}
$$

using in the last equality the fact that $\left\|x-q^{\prime}\right\|+\|Z-x\| \leq R_{M}$ implies that $X_{M} \notin B(x,\|Z-x\|)$, since

$$
B(x,\|Z-x\|) \subseteq B\left(q^{\prime},\left\|x-q^{\prime}\right\|+\|Z-x\|\right) .
$$

Moreover,

$$
\begin{aligned}
& \mathrm{E}\left(\sum_{i=1}^{N} \mathbf{1}\left[X_{i}=Z(x), R_{M-1}<\left\|x-q^{\prime}\right\|+\left\|X_{i}-x\right\| \leq R_{M}\right] \mid Z\right) \\
& =\mathrm{E}\left(\sum _ { i = 1 } ^ { N } \mathbf { 1 } \left[\left[\left(\Psi \backslash\left\{X_{i}\right\}\right) \cup\left\{Z, X_{M}\right\}\right] \cap B\left(x,\left\|X_{i}-x\right\|\right)=\varnothing,\right.\right. \\
& \left(\Psi \backslash\left\{X_{i}\right\}\right) \cup\{Z\} \subset B\left(q^{\prime},\left\|x-q^{\prime}\right\|+\left\|X_{i}-x\right\|\right), \\
& \left.\left.\left\|x-q^{\prime}\right\|+\left\|X_{i}-x\right\| \leq R_{M}\right] \mid Z\right) \\
& =\rho \int \mathbf{1}\left[y \in B\left(q^{\prime}, T+l\right) \backslash B(q, T),\right. \\
& \left.Z \in B\left(q^{\prime},\left\|x-q^{\prime}\right\|+\|y-x\|\right) \backslash B(x,\|y-x\|)\right] \\
& \times \mathrm{P}\left(\left\|x-q^{\prime}\right\|+\|y-x\| \leq R_{M}, X_{M} \notin B(x,\|y-x\|) \mid Z\right) \\
& \times \mathrm{P}\left(\Psi \subset B\left(q^{\prime},\left\|x-q^{\prime}\right\|+\|y-x\|\right) \backslash B(x,\|y-x\|) \mid Z\right) \mathrm{d} y \\
& =\rho \int \mathbf{1}\left[y \in B\left(q^{\prime}, T+l\right) \backslash B(q, T)\right. \text {, } \\
& \left.Z \in B\left(q^{\prime},\left\|x-q^{\prime}\right\|+\|y-x\|\right) \backslash B(x,\|y-x\|)\right] \\
& \times p_{1}(x, y \mid Z) p_{2}(x, y \mid Z) \mathrm{d} y \text {, }
\end{aligned}
$$

using in the second equality the Slivnyak-Mecke formula for the Poisson process $\Phi \backslash\{Z\}$ conditional on $Z$ [2], [7] (or see Theorem 3.2 of [4]), and using in the last equality a similar argument as when we obtained the last equality in (23). Finally, from (22)-(24), we obtain (17). 


\subsection{Equivariance and scaling properties}

The following equivariance property (25) and scaling property (26) simplify things when considering the distribution of $\mathcal{R}$ and in particular the distribution of $V$.

For any number $s, d \times d$ matrix $\mathcal{O}$, and set $A \subseteq \mathbb{R}^{d}$, define $s A=\{s a: a \in A\}, A+q^{\prime}=$ $\left\{a+q^{\prime}: a \in A\right\}$, and $\mathcal{O} A=\{\mathcal{O} a: a \in A\}$. To stress the dependence of $\left(\Phi, q, q^{\prime}\right)$, write $\mathcal{R}=\mathcal{R}\left(\Phi, q, q^{\prime}\right)$ and $V=V\left(\Phi, q, q^{\prime}\right)$. Let $\mathcal{R}(\Phi, l)$ and $V(\Phi, l)$ denote the cases of $\mathcal{R}$ and $V$ when $q^{\prime}=o$ and $q=u$, where the first coordinate of $u$ is $l>0$ and the remaining coordinates are 0. Furthermore, let ' $\sim$ ' mean 'is distributed as'. Then, if $q-q^{\prime}=\mathcal{O} u$ and $\mathcal{O}$ is an orthonormal matrix,

$$
\mathcal{R}\left(\Phi, q, q^{\prime}\right) \sim \mathcal{O} \mathcal{R}(\Phi, l)+q^{\prime}, \quad V\left(\Phi, q, q^{\prime}\right) \sim V(\Phi, l),
$$

since $\mathcal{O}^{\top}\left(\Phi-q^{\prime}\right) \sim \Phi$, where $\mathcal{O}^{\top}$ is the transpose of $\mathcal{O}$. Therefore, without any loss of generality, we can assume that $q^{\prime}=o$ and $q=u$.

Moreover, writing $\Phi=\Phi_{\rho}$ for the Poisson process on $\mathbb{R}^{d}$ with intensity $\rho>0$, we can make the coupling $\Phi_{\rho}=\rho^{-1 / d} \Phi_{1}$. Then

$$
\mathcal{R}\left(\Phi_{\rho}, l\right)=l \mathcal{R}\left(\Phi_{\rho l^{d}}, 1\right)=\rho^{-1 / d} \mathcal{R}\left(\Phi_{1}, \rho^{1 / d} l\right)
$$

and

$$
V\left(\Phi_{\rho}, l\right)=l^{d} V\left(\Phi_{\rho l^{d}}, 1\right)=\rho^{-1} V\left(\Phi_{1}, \rho^{1 / d} l\right),
$$

so, for the volume of the privacy region, it boils down to considering the distribution of either $V\left(\Phi_{\rho}, 1\right)$ for $\rho>0$ (the case with $l=1$ ) or $V\left(\Phi_{1}, l\right)$ for $l>0$ (the case with $\rho=1$ ).

\subsection{Numerical results}

In this section we discuss some numerical results for $p(x)$ and $\mathrm{E}(V)$.

Recall that $p(x)$ is the probability that $x$ belongs to the inferred privacy region. Clearly, $p(q)=1$ since $q \in \mathcal{R}$. For $x \neq q$ and fixed $l$, both $p(x)$ and $\mathrm{E}(V)$ approach 0 as $\rho$ tends to $\infty$. This follows by combining (13)-(15) and (16)-(17), and it is in accordance with the fact that, as $\rho \rightarrow \infty$, then $\|Z-q\| \rightarrow 0$ almost surely, and so $\mathcal{R}$ tends to the empty set. It is interesting to study how $p(x)$ varies as a function of $x$ when $d=2$. In Figure 3 we plot the contours of $p(x)$ when $\rho=1$ and $l=1$. Observe that contours with high $p(x)$ are located close to the point $q=(1,0)$. Contours with low $p(x)$ appear as circle-like shapes, with centre $q^{\prime}=(0,0)$ and in pairs, with radii below 1.0 and above 1.0, respectively. Figure 4 shows the contours of $p(x)$ when $\rho=10$ and $l=1$. This function resembles that of Figure 3, except that the region with high $p(x)$ is shrinked significantly.

Two methods are employed to evaluate $\mathrm{E}(V)$ when $d=2$. Again, we take $q^{\prime}=(0,0)$ and $q=(l, 0)$. The first method is Monte Carlo, which executes 10000 instances of the conventional radial simulation algorithm from [6] for generating a stationary Poisson process until the termination time $M$ is determined. For each simulation, we estimate the area of $V$ by using a $100 \times 100$ square grid over the domain $\left[-R_{M}, R_{M}\right]^{2}$ which contains $\mathcal{R}$. The value of $\mathrm{E}(V)$ is then estimated by the average area obtained from all simulations. The second method is numerical integration, using the following setting when $l=1$ and $\rho=$ $1,2,5,10,20,50,100$.

- The integral in (17) is computed using a $100 \times 100$ square grid in the region $[-(t+l)$, $(t+l)]^{2}$. All possible points $y$ such that the indicator function in (17) is equal to 1 must be located inside such a region. 


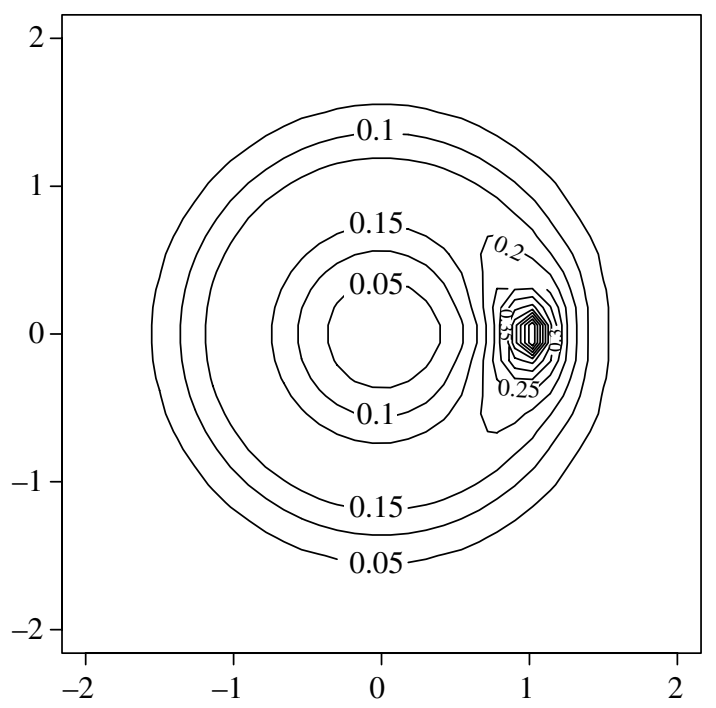

Figure 3: Contours of $p(x)$, when $\rho=1, l=1$, and $d=2$.

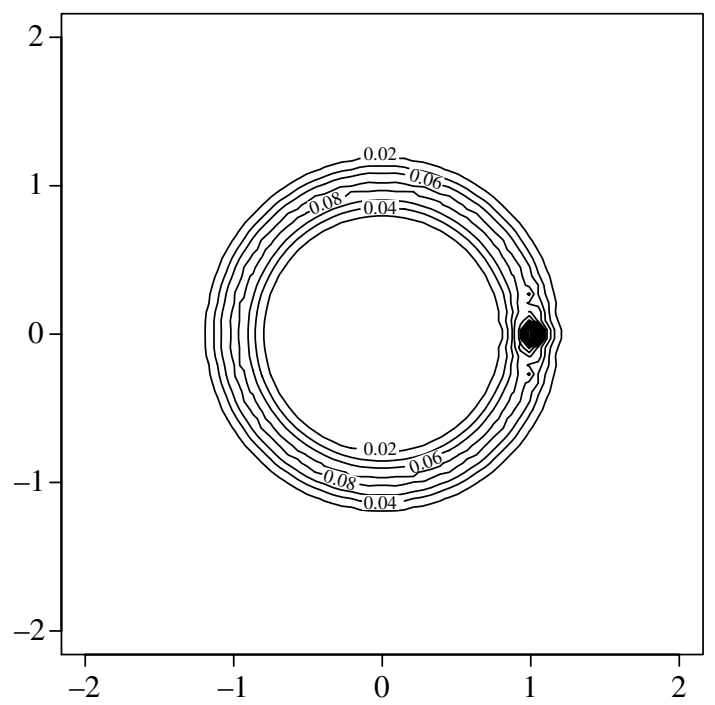

FIGURE 4: Contours of $p(x)$, when $\rho=10, l=1$, and $d=2$.

- The integral in $p(x)=\int p(x \mid z) f(z) \mathrm{d} z$ is computed using a $100 \times 100$ square grid in the region $[-3 l, 3 l]^{2}$. In fact, $p(x)$ is effectively 0 outside this region and seemingly only limited value is lost even though the full space $\mathbb{R}^{2}$ is not used as the domain for numeric integration.

- For $\rho=1,2,5,10,20,50$, the integral in $\mathrm{E}(V)=\int p(x) \mathrm{d} x$ is computed using a $100 \times$ 100 square grid in the region $[-3 l, 3 l]^{2}$. As above, only limited value is lost when $[-3 l, 3 l]^{2}$ is used as the bounding region. For $\rho=100$, after first using the same method and comparing with the Monte Carlo estimate, we found it appropriate to use 


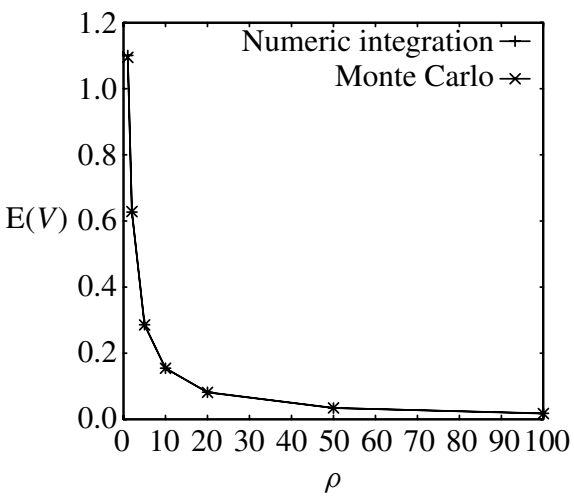

(a) $\mathrm{E}(V)$ versus $\rho$ when $l=1$

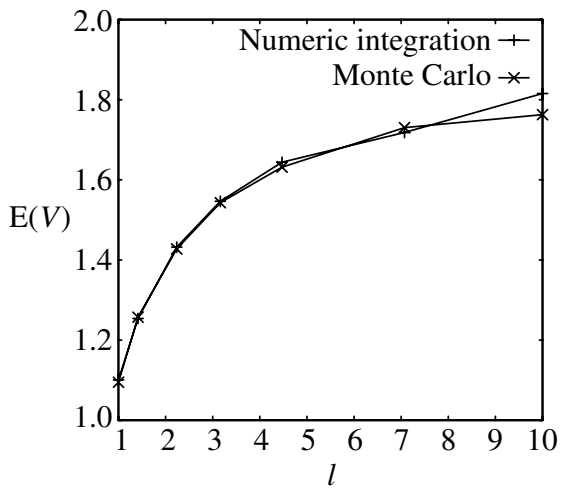

(b) $\mathrm{E}(V)$ versus $l$ when $\rho=1$

FIGURE 5: Evaluation of $\mathrm{E}(V)$.

polar coordinates $(\theta, r)$ for $x$ and a $100 \times 100$ square grid for $(\theta, r)$ in the domain $[0,2 \pi) \times[0.75,1.25)$. Outside this domain $p(x)$ is almost 0 .

Figure 5(a) shows $\mathrm{E}(V)$ as a function of $\rho$ when $l=1$ and $d=2$. Observe that the value obtained by numerical integration is close to the corresponding value obtained by Monte Carlo. For $\rho=100$, the difference between the values obtained by numerical integration and Monte Carlo becomes more visible (the estimates of $\mathrm{E}(V)$ when $(\rho, l)=(100,1)$, obtained using a square grid for respective $x$ and polar coordinates for $x$, are respectively 0.0160 and 0.0182 as compared to the Monte Carlo estimate 0.0176). Figure 5(a) suggests that $\mathrm{E}(V)$ is a strictly decreasing convex function of $\rho$.

In Figure 5(b) we plot $\mathrm{E}(V)$ as a function of $l$ when $\rho=1$ and $d=2$. This plot is just obtained from the results in Figure 5(a) using (26), i.e. the fact that $V\left(\Phi_{1}, l\right) \sim l^{d} V\left(\Phi_{l^{d}}, 1\right)$. Note that $\mathrm{E}(V)$ appears to be an increasing concave function of $l$.

\section{Acknowledgements}

An anonymous referee and the coordinating editor of SGSA are acknowledged for helpful comments. Jesper Møller was supported by the Danish Natural Science Research Council, grants 272-06-0442 and 09-072331, 'Point process modelling and statistical inference', and by the Centre for Stochastic Geometry and Advanced Bioimaging, funded by a grant from the Villum Foundation. Man Lung Yiu was supported by ICRG grants A-PJ79 and G-U807 from the Hong Kong Polytechnic University.

\section{References}

[1] Kingman, J. F. C. (1993). Poisson Processes. Clarendon Press, Oxford, New York.

[2] Mecke, J. (1967). Stationäre zufällige Maße auf lokalkompakten Abelschen Gruppen. Z. Wahrscheinlichkeitsth. 9, 36-58.

[3] Møller, J. (1994). Lectures on Random Voronoi Tessellations (Lecture Notes Statist. 87), Springer, New York.

[4] Møller, J. And WaAgepetersen, R. P. (2004). Statistical Inference and Simulation for Spatial Point Processes (Monogr. Statist. Appl. Prob. 100). Chapman and Hall/CRC, Boca Raton, FL.

[5] Okabe, A., Boots, B., Sugihara, K. And Chiu, S. N. (2000). Spatial Tessellations: Concepts and Applications of Voronoi Diagrams, 2nd edn. John Wiley, Chichester.

[6] Quine, M. P. and Watson, D. F. (1984). Radial generation of n-dimensional Poisson processes. J. Appl. Prob. 21, 548-557. 
[7] SlivnyaK, I. M. (1962). Some properties of stationary flows of homogeneous random events. Teor. Veroyat. Primen. 7, 347-352. English translation: Theory Prob. Appl. 7, 336-341.

[8] Stoyan, D., Kendall, W. S. And Mecke, J. (1995). Stochastic Geometry and Its Applications, 2nd edn. John Wiley, Chichester.

[9] Yiu, M. L., Jensen, C. S., Huang, X. And Lu, H. (2008). SpaceTwist: Managing the trade-offs among location privacy, query performance, and query accuracy in mobile services. In Proc. 24th IEEE Internat. Conf. Data Engineering, eds M. Castellanos, A. P. Buchmann and K. Ramamritham, IEEE Computer Society, Los Alamitos, CA, pp. 366-375. 